\title{
Data-Driven Learning-Based Fault Tolerant Stability Analysis
}

\author{
Lei Ge ${ }^{1}$ and Shun Chen (D) $^{2}$ \\ ${ }^{1}$ School of Finance, Southwestern University of Finance and Economics, Chengdu, Sichuan, China \\ ${ }^{2}$ School of Economics, Huazhong University of Science and Technology, Wuhan, Hubei, China \\ Correspondence should be addressed to Shun Chen; shun6266@gmail.com
}

Received 20 December 2019; Accepted 16 March 2020; Published 9 April 2020

Guest Editor: Viet-Thanh Pham

Copyright (c) 2020 Lei Ge and Shun Chen. This is an open access article distributed under the Creative Commons Attribution License, which permits unrestricted use, distribution, and reproduction in any medium, provided the original work is properly cited.

\begin{abstract}
In this paper, a new data-driven learning method is investigated based on the dynamical data of the system. A regularized regression wavelet (RRW) approach is proposed to optimize the learning result for the system fault. Based on the optimizing results, a fault tolerant stability scheme is given. Then, the efficiency of the proposed technique is verified by a vertical take-off and landing (VTOL) aircraft stability example.
\end{abstract}

\section{Introduction}

The analysis of the data-driven learning method in this paper aims at developing a reliable learning algorithm and applying the method to practical engineering systems. Specifically, we will show how the proposed data-driven learning technique is applied to the dynamical system.

Stability is a fundamental property in modern engineering systems. How to stabilize a system is well studied in the past decades. Plenty of stability controllers are proposed, for example, variable structure control [1], fuzzy control [2], sliding mode control [3], and adaptive neural control [4]. Various undesirable effects are always existed in many engineering applications, such as time-delay [4], fault [5, 6], and uncertainty [7]. In most cases, plenty of these effects can be observed by available measurement data; however in some cases, they are not easily described by existing mathematical models. In particular, these data contain meaningful features of the engineering applications. It is highly desirable to develop new techniques to learn the system data and generate a reliable algorithm based on the real data to cope with those fast changes in the complex systems directly. In this paper, we developed a learning model to address the issue of stabilizing dynamical systems from a different prospective.

During the last decade, data-driven innovation has become a hot topic across different research sectors and provides new challenges associated with various network design, such as sensor networks and telecommunication networks. The network data constitute infrastructural information that could be used in many ways to produce different products and services. It provides additional information over the limitation of traditional system models and also enables creation of knowledge that is crucial for a new design. Recently, there are some data-driven control research studies available in the literature. For example, sampled data stabilization techniques for the T-S fuzzy system were proposed in [8]. A recurrent neural networkbased data-driven control was constructed for the steadystate analysis in [9]. In addition, a lot of research studies have been undertaken to study the data-driven methods in industrial processes, see [10] for a survey. As one may know, the engineering systems are generally operated under different industrial environments. The classic model-based approach could be difficult to stabilize a real system in these types of applications. Hence, one may consider the datadriven approach to analyze the stability of the engineering system.

In particular, faulty issues in highly complexity dynamical systems always exist and are strongly reflected in the collected data. It has certainly a great effect on the safety requirement, and thus the fault reconstruction problem is one of the main concerns in the distributed network control design. The fault reconstruction problem was studied for sensor networks in 
[11], and the results were used for the room temperature monitoring application. Recently, the fault tolerant consensus issue was researched in multiagent systems considering uncertainty in [12]. Data-driven methods for monitoring and fault diagnosis on the benchmark Tennessee Eastman process were investigated in [13].

In this paper, we propose a constructive data learning approach to analyze the effect of the fault. In addition, based on the learning result, the fault tolerant stability of the dynamical system is well studied. The method used in this paper is based on the RRW technique. It should be pointed out that the wavelet functions are used for nonlinear transformation function. Incorporating the time-frequency localization properties of wavelets, it has a strong learning ability for complex nonlinear system modelling. Especially, the wavelet approach has the advantage of dealing with rapid changes of data [14-16]. Inspired by the approach of multiresolution analysis, this paper provides a new datadriven approach for studying fault tolerant stability by applying this powerful wavelet tool. A detailed mathematical approach is constructed to deal with the effect of faults. In this paper, we aim to use the collected data from the dynamical system to stabilize the system. The novelties and advanced features of this paper are as follows:

(1) Compared with the existing stability problem, this paper addresses the fault effect in the dynamical system. In addition, the designed technique used in this paper is a data-driven learning method which is based on a regularized regression wavelet neural network.

(2) It is worth noting that most of the aforementioned literature is about data-driven stability analysis without considering to use a wavelet neural network. In this paper, we use the wavelet neural networkbased learning control to stabilize the dynamical system which has the advantage of dealing with rapid changes of the sensor data and fault.

(3) A detailed mathematical calculation for the reconstruction of the fault in this paper has been given, which potentially contributes to the real-world application of this paper.

The remainder of this paper is organized as follows: in Section 2, some preliminaries are proposed. In Section 3, data-driven fault tolerant stability is analyzed. In Section 4, a VTOL aircraft stability problem is analyzed to illustrate the effectiveness of the theoretical results. The final section concludes this paper.

\section{Preliminaries}

We consider a dynamical system described by the state equation:

$$
\dot{x}(t)=A x(t)+B(u(t)+\bar{g}(x(t))),
$$

where $x(t) \in \mathscr{R}^{n}$ is the state of the system, $\bar{g}(x(t)) \in \mathscr{R}^{q}$ is the fault, $u(t) \in \mathscr{R}^{q}$ is the actuator control input, and $A \in \mathscr{R}^{n \times n}$ and $B \in \mathscr{R}^{n \times q}$ are the known system parameters.
The sensor output of this system is taken to be the same as the state vector.

As is well known, for the fault $\bar{g}(x(t))$, the common assumption is that the uncertainty is norm bounded by a constant $[12,17]$. In classical fault tolerant analysis for a dynamical system, one may use adaptive control to stabilize those faulty systems, see $[12,17]$. In addition, the aforementioned fault assumptions in literature mostly require the fault to be differential, see [11] and the reference therein. However, due to the unpredictability of the fault, one cannot estimate the bound of the fault $\bar{g}(x(t))$, not to mention the differential requirement of the fault. In this paper, we released these assumptions by using a data-driven learning method based on the least-square regularized regression approach.

Before we propose the main results, we first focus on the initial data collecting procedure. Firstly, the system is assumed to be normal, i.e., this system is without fault. It is known that, to stabilize system (1) without fault, that is, to stabilize the following system:

$$
\dot{x}(t)=A x(t)+B u(t) .
$$

One can define the feedback control as $u(k)=-K x(k)$, with the matrix $A-B K$ Hurwitz. Then, based on this construction, we initially collected the data with the feedback control $u(k)=-K x(k)$. However, it should be pointed out that while the fault is considered, system (1) may be unstable. Next, based on the data generated by the faulty system, we will identify the fault by using the following results.

We let $z(t)$ be the regression vector in the regression model, $z(t)=x(t)$. For the state sensor output $x(t)$, we calculate the derivative of state $x(t)$ numerically, i.e., $\dot{x}(t)=$ $(x(t+h)-x(t)) / h$ mathematically, where $h$ is the numerically sampling period. Then, we can obtain $y(t)$ as $\dot{x}(t)-$ $A x(t)-B u(t)$ which is assumed to be the data output. We use this relation to evaluate data samples as $\{z(t), y(t)\}_{t=0}^{T}$.

\section{Main Results}

The data-driven learning problem is formulated as

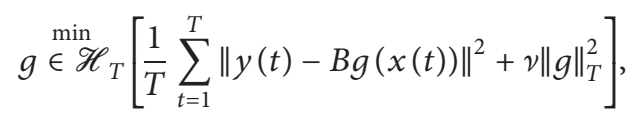

where $g=\left[g_{1}, g_{2}, \ldots, g_{q}\right]^{T},\left\|g_{i}\right\|^{2}=\left\langle g_{i}, g_{i}\right\rangle_{\mathscr{H}_{T}}$, and the regularization constant $\nu$ is used to make a trade-off between the empirical approximation error and the complexity of the model. The solution of the above optimization problem is expressed as

$$
g_{i}\left(x_{v}\right)=\sum_{t=1}^{T} c_{g_{i}} K\left(x_{v}, x(t)\right)
$$

Next, we shall consider the nonlinear function approximation method in this paper. The reproducing kernel Hilbert space (RKHS) is defined to be the closure of linear span of a set of functions, with the kernel functions used for approximation be nonlinear functions. In this paper, we use Morlet WNN kernel: 


$$
K_{\mathrm{WNN}}\left(x, x^{\prime}\right)=\prod_{i=1}^{n} \cos \left(\omega_{0} \frac{\left(x_{i}-x_{i}^{\prime}\right)}{a_{i}}\right) \exp \left(-\frac{\left(x_{i}-x_{i}^{\prime}\right)^{2}}{2 a_{i}^{2}}\right) .
$$

The Morlet wavelet is a wavelet composed of a complex exponential (carrier) multiplied by a Gaussian window (envelope). The parameter $\omega_{0}>0$ represents the dilation, and the parameter $a_{i}>0$ represents translation. These two parameters can be adjusted by users. One can find the theoretical analysis for optimal dilation and translation parameters selection in [15]. Then, the RKHS is a space of nonlinear functions and has good approximation performance. We solve the above optimization problem which is given by the following theorem.

Theorem 1. For the optimization problem (3), the solution is obtained by the following formula:

$$
g_{j}(x)=\sum_{t=1}^{T} l_{j t} K_{\mathrm{WNN}}(x(t), x),
$$

where $l_{j t}$ is the solution of the following equation:

$$
\left(T \nu+\left(I_{T} \otimes B^{T} B\right) \bar{G}\left(I_{q} \otimes T_{m}\right) \bar{L}\right) l=\left(I_{T} \otimes B^{T}\right) y,
$$

where

$$
T_{m}=\left(T_{m i j}\right)_{T \times T}, T_{m i j}=K_{\mathrm{WNN}}(x(j), x(i)),
$$

and $\bar{G}$ and $\bar{L}$ satisfy $g_{T}=\bar{G} \bar{g}_{T}$ and $\bar{l}=\bar{L} l$, respectively, with

$$
\begin{aligned}
\bar{g}_{j T} & =\left[g_{j}(x(1)), g_{j}(x(2)), \ldots, g_{j}(x(T))\right]^{T} \in \mathscr{R}^{T}, \\
\bar{g}_{T} & =\left[\bar{g}_{1 T}, \bar{g}_{2 T}, \ldots, \bar{g}_{q T}\right]^{T} \in \mathscr{R}^{T q}, \\
g_{t T} & =\left[g_{1}(x(t)), g_{2}(x(t)), \ldots, g_{q}(x(t))\right]^{T} \in \mathscr{R}^{q}, \\
g_{T} & =\left[g_{1 T}, g_{2 T}, \ldots, g_{T T}\right]^{T} \in \mathscr{R}^{T q}, \\
\bar{l}_{j} & =\left[l_{j 1}, l_{j 2}, \ldots, l_{j T}\right]^{T} \in \mathscr{R}^{T}, \\
\bar{l} & =\left[\bar{l}_{1}, \bar{l}_{2}, \ldots, \bar{l}_{q}\right]^{T} \in \mathscr{R}^{T q}, \\
l_{t} & =\left[l_{1 t}, l_{2 t}, \ldots, l_{q t}\right]^{T} \in \mathscr{R}^{q}, \\
l & =\left[l_{1}, l_{2}, \ldots, l_{T}\right]^{T} \in \mathscr{R}^{T q} .
\end{aligned}
$$

Proof. Introduce an orthonormal wavelet $\left\{\phi_{p, q}\right\}$ in $L_{\rho}^{2}(X)$. Their corresponding eigenvalues are $\left\{\lambda_{p, q}\right\}$. Then, we have $g_{i}=$ $\sum_{p, q=-\infty}^{\infty} c_{g_{i} p, q} \phi_{p, q}$ for any $g_{i} \in \mathscr{H}_{\mathrm{WNN}}$. The parameters $c_{g_{i} p, q}$ are to be chosen to minimize the objective function (3).

Let

$$
\begin{aligned}
P & =\frac{1}{T} \sum_{t=1}^{T}(y(t)-B g(x(t)))^{T}(y(t)-B g(x(t)))+v\|g\|_{T}^{2} \\
& =\frac{1}{T} \sum_{t=1}^{T} \sum_{i=1}^{n} y_{i}(t)-\sum_{j=1}^{q} b_{i j} g_{j}(x(t))^{2}+\nu\|g\|_{T}^{2} g_{j}(x(t))^{2}+v\|g\|_{T}^{2} .
\end{aligned}
$$

Then, one can obtain

$$
\begin{aligned}
\frac{\partial P}{\partial c_{g_{j} p, q}}= & -\frac{2}{T} \sum_{t=1}^{T} \sum_{i=1}^{n}\left(y_{i}(t)-\sum_{j=1}^{q} b_{i j} g_{j}(x(t))\right) b_{i j} \phi_{p, q}(x(t)) \\
& +2 \frac{\nu c_{g_{j} p, q}}{\lambda_{p, q}} \\
= & -\frac{2}{T} \sum_{t=1}^{T} \sum_{i=1}^{n}\left(b_{i j} y_{i}(t)-b_{i j} \sum_{j=1}^{q} b_{i j} g_{j}(x(t))\right) \phi_{p, q}(x(t)) \\
& +2 \frac{\nu c_{g_{j} p, q}}{\lambda_{p, q} .}
\end{aligned}
$$

Considering the minimum optimization problem, we have

$$
c_{g_{j} p, q}=\lambda_{p, q} \frac{\sum_{t=1}^{T} \sum_{i=1}^{n} b_{i j} y_{i}(t)-b_{i j} \sum_{j=1}^{q} b_{i j} g_{j}(x(t)) \phi_{p, q}(x(t))}{T \nu} .
$$

Using the fact that

$$
K_{\mathrm{WNN}}(x(t), x)=\sum_{p, q=-\infty}^{\infty} \lambda_{p, q} \phi_{p, q}(x(t)) \phi_{p, q}(x),
$$

we can get

$$
\begin{aligned}
g_{j}(x) & =\sum_{p, q=-\infty}^{\infty} c_{g_{j} p, q} \phi_{p, q}(x) \\
& =\sum_{p, q=-\infty}^{\infty} \lambda_{p, q} \frac{\sum_{t=1}^{T} \sum_{i=1}^{n}\left(b_{i j} y_{i}(t)-b_{i j} \sum_{j=1}^{q} b_{i j} g_{j}(x(t))\right) \phi_{p, q}(x(t))}{T \nu} \phi_{p, q}(x) \\
& =\frac{\sum_{t=1}^{T} \sum_{i=1}^{n}\left(b_{i j} y_{i}(t)-b_{i j} \sum_{j=1}^{q} b_{i j} g_{j}(x(t))\right) K_{W N N}(x(t) x)}{T \nu} \\
& =\sum_{t=1}^{T} l_{j t} K_{\mathrm{WNN}}(x(t) x) .
\end{aligned}
$$


From (9),

$$
\begin{aligned}
\bar{g}_{j T} & =T_{m} \bar{l}_{j}, \\
\bar{g}_{T} & =\left(I_{q} \otimes T_{m}\right) \bar{l},
\end{aligned}
$$

we have

$$
\begin{aligned}
T \nu l_{t} & =B^{T} y(t)-B^{T} B g_{t T}, \\
T \nu l & =\left(I_{T} \otimes B^{T}\right) y-\left(I_{T} \otimes B^{T} B\right) g_{T} \\
& =\left(I_{T} \otimes B^{T}\right) y-\left(I_{T} \otimes B^{T} B\right) \bar{G} \bar{g}_{T} \\
& =\left(I_{T} \otimes B^{T}\right) y-\left(I_{T} \otimes B^{T} B\right) \bar{G}\left(I_{q} \otimes T_{m}\right) \bar{l} \\
& =\left(I_{T} \otimes B^{T}\right) y-\left(I_{T} \otimes B^{T} B\right) \bar{G}\left(I_{q} \otimes T_{m}\right) \bar{L} l .
\end{aligned}
$$

The theorem is obtained.

The above optimization problem leads to the following approximation results:

$$
\min _{g_{i} \in \mathscr{H}_{T}\|B \bar{g}(x(t))-B g(x(t))\| .}
$$

Then, by using the cross-validation method, we can estimate the approximation error $\mathbf{e}$ in (17) which is not exactly known. In practice, define $\varsigma$ as the upper bound of the approximation error which can be computed by the data learning algorithm. In fact, one can find that a good approximation error at $O\left(10^{-3}\right)$ or even a smaller value can be obtained in simulations by using sufficiently large data samples for the learning. To ensure the control design to be robust under faulty data, the upper bound of the approximation error $\varsigma$ is set to be $10 \mathbf{e}$ in our data learning algorithm.
That is, $\|B \bar{g}(x(t))-B g(x(t))\| \leq \varsigma$. This construction is explained based on the following facts: as one may know that the design of the control is based on future system process which is fully unknown. This makes the learning of future fault very difficult. Although the cross-validation method can estimate the error very well, there will still be some differences between the estimated fault and the true fault. That is to say, the learned fault obtained by approximating the trained data can reflect most of the parts of the fault function, but not the exactly true fault. Nevertheless, the parameter $\varsigma$ produced from training data can provide a good result and is used to estimate the approximate error.

In the following, we will give a theorem to design the fault tolerant control based on the approximation result.

Theorem 2. Considering the system with the fault given in (1), the system will be stabilized under the following control:

$$
u(t)=B^{T} P x(t)-\operatorname{sign}\left(B^{T} P x(t)\right) \varsigma-g(x(t)),
$$

where $P>0$ is the unique solution to the algebraic Riccati equation (ARE):

$$
A^{T} P+P A-P B B^{T} P+I=0 .
$$

Proof. Introduce the following Lyapunov candidate:

$$
V(t)=x^{T}(t) P x(t) .
$$

Then, the derivative of $V(t)$ satisfies

$$
\begin{aligned}
\dot{V}(t)= & 2\left(A x t(t) n+q B h\left(B^{T} P x(t)-\operatorname{sign}\left(B^{T} P x(t)\right) \varsigma-g(x(t))+\bar{g}(x(t), k)\right)\right)^{T} P x(t) \\
= & x^{T}(t)\left(A^{T} P+P A\right) x(t)+x^{T}(t) P B B^{T} P x(t) \\
& -\varsigma \operatorname{sign}\left(B^{T} P x(t)\right) B^{T} P x(t)+e_{g}^{T}(t) B B^{T} P x(t) \\
\leq & x^{T}(t)\left(A^{T} P+P A+P B B^{T} P\right) x(t) .
\end{aligned}
$$

From (19), the theorem is obtained.

Remark 1. In this paper, the data-driven learning method is proposed to identify the system fault. It should be pointed out that there exist some problems which remain to be solved: (i) since the data are collected for learning, it is well known that identification efficiency can be improved as more data samples are collected. The amount of data to be collected to improve the efficiency of the algorithm is still an open problem; (ii) from (3), one can find that there exists a regularization constant, $v$ in (3), to reduce the model complexity and the learning error. The constant is always case dependent; (iii) the choice of the dilation $\omega_{0}$ and violation $a_{i}$ in the Morlet WNN kernel is also case dependent. One can refer to WNN for learning function in $[14,15]$.
Remark 2. In this paper, the data-driven learning method is given to analyze the system fault. This is different from the classic fault tolerant analysis in the networked system. Classically, the fault analysis used the model-based approach, see [17-19] and the reference therein, in which they solved the fault tolerant problem in a system basis. However, in this paper, we analyze the fault fully based on the historical data, and some useful data-based results are obtained. This makes a new insight in applying data learning strategies in the fault research area.

Remark 3. The results obtained in this paper aim to solve the stability problem in a dynamical system considering fault by using the WNN-based method. Through Theorem 1, we can obtain the detailed formula for the approximation of the system fault effect in (1). One can refer to (17) for details. In 
addition, with the given equations (6)-(9), one can easily calculate the mathematical form of the control in (18). All these procedures contribute to the application of the obtained results in real-world engineering problems.

\section{Application}

In this section, the VTOL aircraft stability problem is proposed to verify the method in Theorem 2 . One typical VTOL aircraft model can be found in Yakovlev Yak-38 which is a Soviet Navy VTOL aircraft intended for their light carriers, cargo ships, and capital ships. The dynamical system is described by

$$
\dot{x}(t)=A x(t)+B(u(t)+\bar{g}(x(t))) .
$$

The parameter matrices $A$ and $B$ are given as follows:

$$
\begin{aligned}
A & =\left(\begin{array}{cccc}
-0.0366 & 0.0271 & 0.0188 & -0.4555 \\
0.0482 & -1.01 & 0.0024 & -4.0208 \\
0.1002 & 0.3681 & -0.707 & 1.42 \\
0 & 0 & 1 & 0
\end{array}\right), \\
B & =\left(\begin{array}{cc}
0.4422 & 0.1761 \\
3.5446 & -7.5922 \\
-5.52 & 4.49 \\
0 & 0
\end{array}\right) .
\end{aligned}
$$

Based on the proposed method, we use the control $u(t)=-B^{T} P x(t)$ to collect data in $[5 \mathrm{~s}, 10 \mathrm{~s}]$, where

$$
P=\left(\begin{array}{cccc}
2.2932 & 0.1056 & 0.0837 & -0.9103 \\
0.1056 & 0.1940 & 0.1357 & -0.0156 \\
0.0837 & 0.1357 & 0.2681 & 0.1683 \\
-0.9103 & -0.0156 & 0.1683 & 1.8079
\end{array}\right) .
$$

The fault considered in the first 10 seconds is given by

$$
\bar{g}(x(t))=\left(\begin{array}{l}
\sin \left(x_{1}(t)\right)-2 \sin \left(x_{3}(t)\right) \\
2 \sin \left(x_{2}(t)\right)-\sin \left(x_{4}(t)\right)
\end{array}\right) .
$$

Then, we can learn the fault effect $B g(x(t))$ numerically through the optimization (3) approach result in (7), see Figure 1. In Figure 1, it can be found that the dashed lines and solid lines are very close which means that the optimization problem in (3) is well solved by using the proposed Theorem 1. The fault considered in the system is given in (25). For illustration purpose, the parameter $v$ in (3) is 0.8 . The dilation and violation constants in (5) are set to be $\omega_{0}=0.5$ and $a_{1}=a_{2}=a_{3}=a_{4}=2$. The control proposed in (18) is applied at $t=10 \mathrm{~s}$ with the $\varsigma$ equal to 0.3 . The trajectory of the system is shown in Figure 2. In Figure 2, we aim to show the effectiveness of the proposed controller in (18). From the trajectory of $x(t)$ in Figure 2, one can find that the system is stabilized since the value of $x(t)$ goes to zero. One can see that the chattering phenomena is shown in this figure. This is due to the fact that the control (18) is based on the function sign. One may use other functions to replace

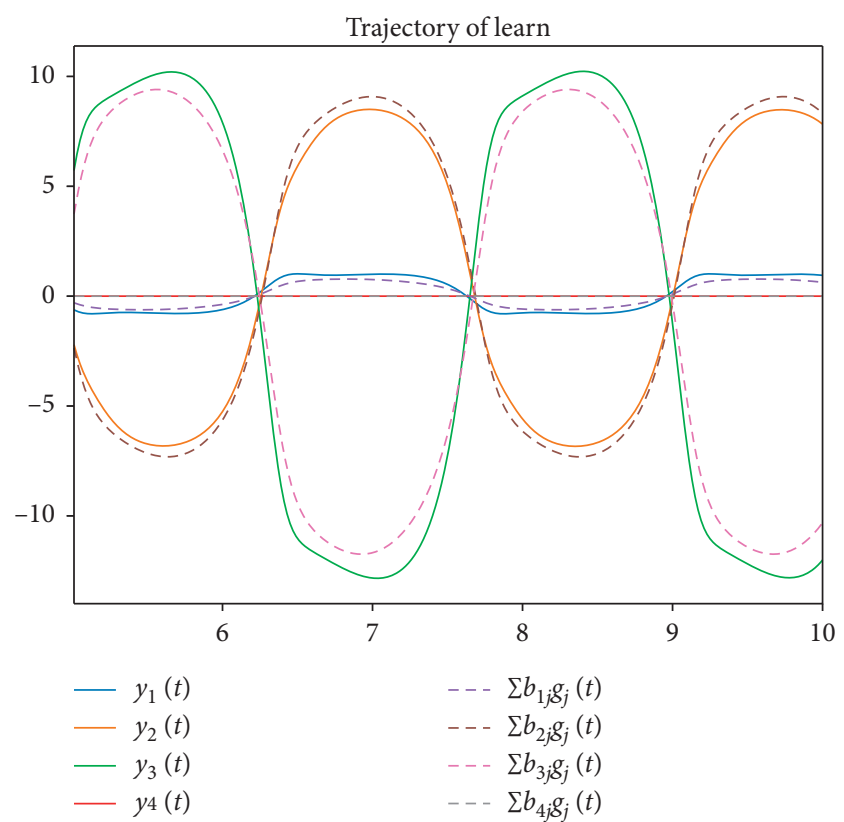

FIGURE 1: Learning result (this figure shows the learning result in (6) which is based on the optimization problem in (3). $\sum b_{i j} g_{j}, i=$ $\{1,2,3,4\}$ represents $\sum_{j=1}^{q} b_{i j} g_{j}, i=\{1,2,3,4\}$, see (10) for the detail calculation).

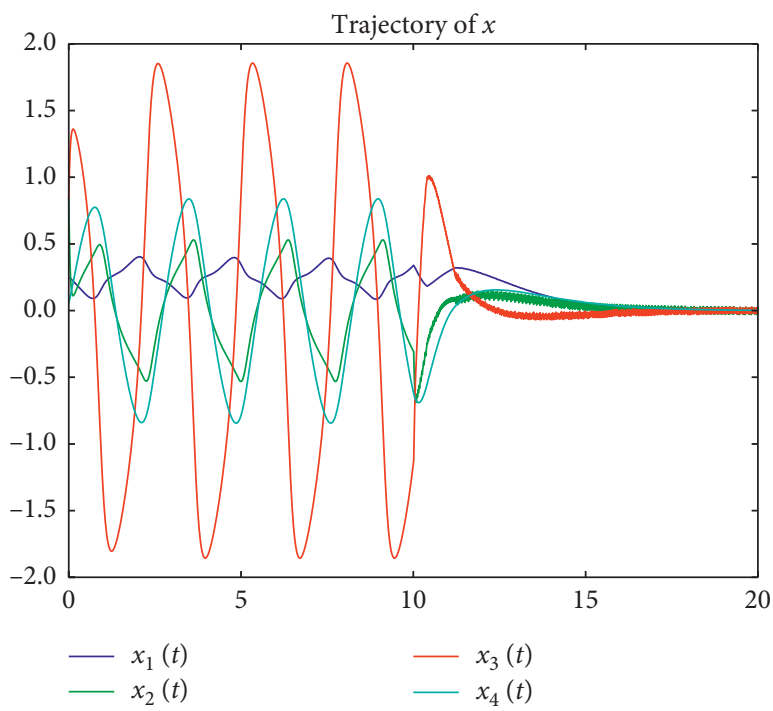

Figure 2: Trajectory of the state (this figure shows the trajectory of the dynamical system (22) with the given fault in (25) by using the proposed learning-based control in (18)).

the sign, which may lead to a bounded domain of the trajectory, see [12].

Next, we will consider different fault effects in [0 s, $50 \mathrm{~s}]$. The fault of the aircraft in $[0 \mathrm{~s}, 20 \mathrm{~s}]$ is

$$
\bar{g}(x(t))=\left(\begin{array}{c}
\sin \left(x_{2}(t)\right)-2 \sin \left(x_{3}(t)\right)+\sin \left(x_{4}(t)\right) \\
\sin \left(x_{1}(t)\right)-2 \sin \left(x_{2}(t)\right)
\end{array}\right) .
$$

The fault of this system in [20 s, $50 \mathrm{~s}]$ is 


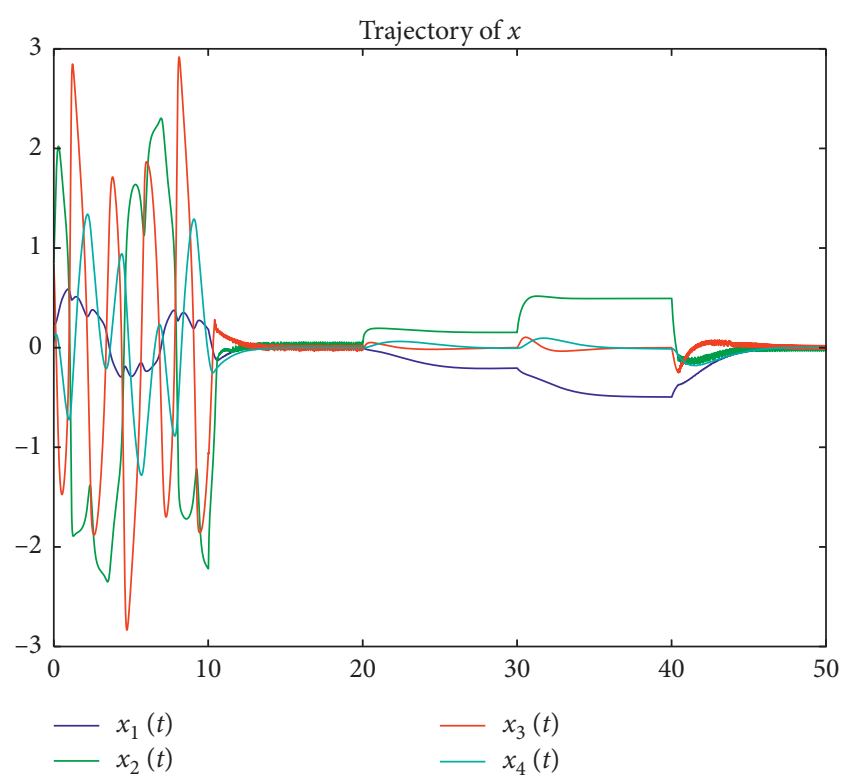

FIgURE 3: Trajectory of the state (this figure shows the trajectory of the dynamical system (22) with the given fault in (26) and (27) by using the proposed learning-based control in (18)).

$$
\bar{g}(x(t))=\left(\begin{array}{l}
-0.5 \\
-0.5
\end{array}\right) .
$$

We analyze the whole process in the following sessions:

Session 1: observation time interval: [20 s, $35 \mathrm{~s}$;

Session 2: data collection time intervals: [5s, $10 \mathrm{~s}]$ and [35 s, $40 \mathrm{~s}$ ];

Session 3: data-driven learning control time intervals: $[10 \mathrm{~s}, 20 \mathrm{~s}]$ and $[40 \mathrm{~s}, 50 \mathrm{~s}]$.

During the first 5 seconds, the operation system data are collected. One may observe that the system is not stable from the collected data. Then, we make the judgement that the system is faulty. In the next 5 seconds, we collect the data and use the proposed learning technique in Theorem 1 to identify the fault. From the data learned results, one may use the control in (18) to stabilize the system. From Figure 3, one can see that, after the controller is applied, the system is stabilized.

Considering the changing fault at $20 \mathrm{~s}$, it can be observed that the previous control is failed to stabilize the system in the time interval [ $20 \mathrm{~s}, 30 \mathrm{~s}$ ]. This makes us to remove the aforementioned controller and repeat the observation-collection learning progress. The details are given as following steps: (1) collect data in [35 s, $40 \mathrm{~s}]$; (2) use the proposed learning technique to identify the fault; (3) apply the newly identified results into the controller (18).

From Figure 3, one can see that, after the controller is applied at $40 \mathrm{~s}$, the system goes to the stability state during [ $40 \mathrm{~s} 50 \mathrm{~s}$ ]. In Figure 3, we consider different fault effects in different time intervals during the system operating. One can find that if two faults are observed at different time, we can always apply the proposed controller in (18) by using the collected data to stabilize the system in the time intervals [10 s, $20 \mathrm{~s}]$ and [40 s, $50 \mathrm{~s}$.

\section{Conclusion}

In this paper, fault tolerant stability is given based on datadriven learning techniques. A regularized regression wavelet approach is proposed for the data-based fault identification. The data-driven scheme is investigated under the consideration to minimize the error between the fault effect and data samples. Based on the learning result, data-driven control is proposed to stabilize the system. Finally, a simulation example is exploited to show the effectiveness of the main result.

\section{Data Availability}

The data used to support the findings of this study are available from the corresponding author upon request.

\section{Conflicts of Interest}

The authors declare that they have no conflicts of interest.

\section{Acknowledgments}

This work was supported by the Fundamental Research Funds for the Central Universities (220110004005040120 and 220110001002020043).

\section{References}

[1] B. Paden and S. Sastry, "A calculus for computing Filippov's differential inclusion with application to the variable structure control of robot manipulators," IEEE Transactions on Circuits and Systems, vol. 34, no. 1, pp. 73-82, 1987.

[2] S. Chiu and S. Chand, "Fuzzy controller design and stability analysis for an aircraft model," in Proceedings of the American Control Conference, pp. 821-826, Boston, MA, USA, June 1991.

[3] K. D. Young, V. I. Utkin, and U. Ozguner, "A control engineer's guide to sliding mode control," IEEE Transactions on Control Systems Technology, vol. 7, no. 3, pp. 328-342, 1999.

[4] D. W. C. Ho, J. Li, and Y. Niu, "Adaptive neural control for a class of nonlinearly parametric time-delay systems," IEEE Transactions on Neural Networks, vol. 16, no. 3, pp. 625-635, 2005.

[5] R. Isermann, R. Schwarz, and S. Stolzl, "Fault-tolerant driveby-wire systems," IEEE Control Systems, vol. 22, no. 5, pp. 64-81, 2002.

[6] Z. Chen, Q. Chen, X. He, and M. Sun, "Adaptive finite-time command filtered fault-tolerant control for uncertain spacecraft with prescribed performance," Complexity, vol. 2018, Article ID 4912483, 12 pages, 2018.

[7] Y. Xiong and M. Saif, "Sliding mode observer for nonlinear uncertain systems," IEEE Transactions on Automatic Control, vol. 46, no. 12, pp. 2012-2017, 2001.

[8] H. Gao and T. Chen, "Stabilization of nonlinear systems under variable sampling: a fuzzy control approach," IEEE Transactions on Fuzzy Systems, vol. 15, no. 5, pp. 972-983, 2007.

[9] H. Zhang, L. Cui, X. Zhang, and Y. Luo, "Data-driven robust approximate optimal tracking control for unknown general nonlinear systems using adaptive dynamic programming method," IEEE Transactions on Neural Networks, vol. 22, no. 12, pp. 2226-2236, 2011. 
[10] S. Yin, S. X. Ding, X. Xie, and H. Luo, "A review on basic datadriven approaches for industrial process monitoring," IEEE Transactions on Industrial Electronics, vol. 61, no. 11, pp. 6418-6428, 2014.

[11] S. Chen, D. W. C. Ho, and C. Huang, "Fault reconstruction and state estimator design for distributed sensor networks in multitarget tracking," IEEE Transactions on Industrial Electronics, vol. 62, no. 11, pp. 7091-7102, 2015.

[12] S. Chen, D. W. C. Ho, L. Li, and M. Liu, "Fault-tolerant consensus of multi-agent system with distributed adaptive protocol," IEEE Transactions on Cybernetics, vol. 45, no. 10, pp. 2142-2155, 2015.

[13] S. Yin, S. X. Ding, A. Haghani, H. Hao, and P. Zhang, "A comparison study of basic data-driven fault diagnosis and process monitoring methods on the benchmark Tennessee Eastman process," Journal of Process Control, vol. 22, no. 9, pp. 1567-1581, 2012.

[14] D. W. C. Ho, P.-A. Ping-Au Zhang, and J. Jinhua Xu, "Fuzzy wavelet networks for function learning," IEEE Transactions on Fuzzy Systems, vol. 9, no. 1, pp. 200-211, 2001.

[15] J. Xu and D. W. C. Ho, "A basis selection algorithm for wavelet neural networks," Neurocomputing, vol. 48, no. 1-4, pp. 681-689, 2002.

[16] F. Wu and Y. Zhao, "Least squares support vector machine on Moret Wavelet kernel function," in Proceedings of the 2005 International Conference on Neural Networks and Brain, vol. 1, pp. 327-331, Beijing, China, October 2005.

[17] M. Liu, D. W. C. Ho, and P. Shi, "Adaptive fault-tolerant compensation control for Markovian jump systems with mismatched external disturbance," Automatica, vol. 58, pp. 5-14, 2015.

[18] S. X. Ding, Model-based Fault Diagnosis Techniques: Design Schemes, Algorithms, and Tools, Springer, Berlin, Germany, 2008.

[19] M. Liu and P. Shi, "Sensor fault estimation and tolerant control for Itô stochastic systems with a descriptor sliding mode approach," Automatica, vol. 49, no. 5, pp. 1242-1250, 2013. 